Universidad íntima y sexualidades públicas. La gestión de la identidad en la experiencia

Educación, Lenguaje y Sociedad ISSN 1668-4753 Vol. XIII Nº 13 (Abril 2016) pp. 1-8

\title{
Rafael Blanco
}

Investigador asistente CONICET y del Instituto de Investigaciones Gino Germani (UBA). Docente de la Facultad de Ciencias Sociales de la UBA.

Universidad íntima y sexualidades públicas. La gestión de la identidad en la experiencia estudiantil

Buenos Aires: Miño y Dávila, 2014, 187 pp.

“Dónde están las lesbianas en esta facultad?” (Grafiti en el baño de la Facultad de Psicología).

T a frase citada forma parte del diseño de tapa del

Llibro, en la que se pueden distinguir fotografías de los baños de las facultades en las que fueron escritas. Ese insumo es solo de una de las aristas desde las cuales el libro Universidad íntima y sexualidades públicas. $L a$ gestión de la identidad en la experiencia estudiantil, del investigador argentino Rafael Blanco, propone problematizar las modalidades en las que las expresiones e identidades de género y sexualidad son producidas en las instituciones universitarias.

Esta obra analiza un conjunto de prácticas, espacios y discursos presentes en la experiencia estudiantil que operan organizando las modalidades que adquieren las sexualidades en la universidad, un espacio poco explorado en comparación con otras instituciones educativas. Estas regulaciones se producen, sostiene el autor, según un conjunto de prescripciones y orientaciones presentes en las culturas institucionales de cada facultad.

El libro aquí reseñado se inscribe en el campo de estudios sobre la universidad pública como objeto de 
investigación, campo que habitualmente aborda con más recurrencia ciertas temáticas por sobre otras: tradicionalmente se han privilegiado los análisis vinculados con los cambios de las políticas universitarias, las orientaciones globales en la educación superior y la crisis de las instituciones modernas y con análisis del movimiento estudiantil. Estos estudios permitieron inscribir históricamente a la universidad, así como también interpretarla, en el marco de los cambios que se iban consolidando de manera global; sin embargo el análisis de la normatividad sexo genérica ha estado ausente de sus estudios. De esta manera la misma operación que inscribía a esta institución a nivel macro,

Iba invisibilizando el lugar que los distintos sujetos institucionales (profesores, estudiantes, investigadores, autoridades, personal) ocupan en la producción y reproducción de estas instituciones, sus modos 'de salir adelante' y vivir en ellas, como así también los impactos de estos procesos en la vida cotidiana (Blanco, 2014, p. 29).

Universidad intima y sexualidades públicas se inscribe entonces en un nuevo giro de los estudios sobre la universidad pública en Argentina, recupera la experiencia de los sujetos que participan de ella, su actividad diaria y experiencial, para comprender ciertos fenómenos del presente imbricados en el acontecer universitario.

Sin embargo, la investigación reseñada en este libro no puede circunscribirse únicamente al campo de los estudios sobre la universidad, dado que se nutrió de los debates y escritos en otras tres áreas: los estudios biográficos, sobre género y sobre juventudes.

Es más conocida a aquella tradición atenta a las modulaciones de las identidades en los itinerarios biográficos, perspectiva abordada para los estudios sobre universidad por autores como Sandra Carli (2012) y Pedro 
Krotsch (2014) que observaron en la experiencia estudiantil "una trama heterogénea de acontecimientos que deja su huella en las biografías de los sujetos y que articula, entre múltiples procesos, aquellos relativos a las formas de vivir y manifestar las expresiones e identidades" (Blanco, 2014, p. 26).

Asimismo, desde los estudios sobre juventudes se han señalado los lazos estrechos entre la condición juvenil y la de estudiante: decontruyendo aquella enquistada trama que asocia "lo joven" con cierto carácter biológico del desarrollo e inscribiéndolo sociohistóricamente en un entramado específico de condiciones de vida particulares en el que las instituciones educativas cumplieron en la historia un rol central.

Nos encontramos entonces con tres campos íntimamente entrelazados: los estudios biográficos, sobre universidades y sobre juventudes. Por otro lado, las problemáticas vinculadas con el género y la sexualidad, si bien han ganado relevancia en la investigación social, así como también en el marco de distintas instituciones educativas, no se plasmaron en análisis en el ámbito universitario.

Universidad íntima y sexualidades públicas conforma entonces una obra pionera en su tipo, que se ha permitido incursionar desde otros puntos de vista en objetos de estudios clásicos, identificando dimensiones de análisis hasta hoy no exploradas.

\section{Jóvenes, sexualidades y experiencia universitaria}

Pese a su posible o aparente desencanto, al conocido "declive de la institución" que mencionaba Dubet (2006), a la acompasada dispersión de un tipo de 
sociabilización clásicamente tipificada como "moderna" hacia otras formas de organización, la universidad sigue siendo depositaria de ciertos imaginarios adquiridos luego de siglos de ser atizados. ¿Qué nos dicen sobre ello las experiencias de los sujetos?

En este sentido, las dimensiones posibles de análisis son múltiples. Específicamente refiriéndonos al estudio de Blanco, que consiste en gran parte en la tesis de doctorado en ciencias sociales del autor realizada en la Universidad de Buenos Aires bajo la dirección de Sandra Carli, se interesa en particular por lo que denomina la dimensión sexuada de la experiencia estudiantil universitaria, y enfoca su análisis en la vida cotidiana de los estudiantes que transitan por estas instituciones.

El libro se propone como objetivo una nueva mirada sobre la universidad pública a partir de la indagación de las formas de gestión de la identidad de género y sexualidad que supera la ya consabida propuesta analítica de los estudios sociológicos que indaga la composición de la matrícula universitaria y su creciente feminización, para complejizar la mirada del género y la sexualidad más allá de la dicotomía hombre/mujer.

Con dicho objetivo elabora un conjunto de dimensiones de análisis que comienza con la dimensión sexuada, línea conceptual que articula todo el libro. A partir de allí aborda las culturas institucionales de cada una de las facultades mencionadas, los espacios públicos y privados dentro de la universidad y las dinámicas socio sexuales que se producen en ellos, los modos de sociabilidad y las agendas de las agrupaciones estudiantiles sobre la sexualidad, problematizando los alcances de "lo público" y "lo privado" en la universidad. 


\section{Instituciones y sociablilidades públicas y privadas}

Como se mencionó en la introducción, el abordaje analítico de la relación entre universidad y regulaciones sexo genéricas puede ser llevado a cabo a partir de múltiples aristas; tanto desde el estudio de los currículos, programas y formatos institucionales, como de las normativas y políticas gubernamentales e institucionales de la propia universidad; sin embargo la propuesta del libro es otra.

La obra parte de la concepción de que las universidades son un espacio de gestión de la identidad personal, en cuya lógica se encuentran los modos legítimos de asumir, encarnar y expresar el género y la sexualidad según un conjunto de prescripciones y de orientaciones implícitas. A partir de esta idea el estudio pretende demostrar que la sociabilidad estudiantil está configurada por el modo en que los y las estudiantes se reconocen y son reconocidos como varones, mujeres, trans, heterosexuales, lesbianas y gays y que estas formas de expresarse e identificarse regulan la experiencia estudiantil en distintos acontecimientos cotidianos.

Un primer acercamiento al campo y su consecuente trabajo de indagación de tipo etnográfico habilitó la emergencia de ciertas dimensiones como más significativas para este estudio por sobre otras que incluyeron, por ejemplo, la delimitación del referente empírico de indagación en las facultades de Ciencias Exactas y Naturales [FCEN] y de Psicología de la Universidad Nacional de Buenos Aires [UBA].

La elección por parte del autor no fue azarosa sino que se trata de instituciones que emergieron como significativas para el estudio porque le permitieron trabajar mejor las diferencias entre el vínculo del conocimiento 
impartido en cada una de las facultades y la sexualidad pensada desde la biología o la psicología ¿Qué presencia tienen estos saberes disciplinares en la trama sexogenérica de los estudiantes de la UBA? ¿Cuál es la dimensión performativa del conocimiento en la vida cotidiana? Todo ello sin olvidar las características particulares de cada una de las instituciones; por ejemplo, la Facultad de Psicología cuenta con un porcentaje de estudiantes mujeres del 83,7\%.

Universidad intima y sexualidades públicas se focaliza entonces en el análisis de los procesos normativos que regulan las expresiones e identidades de género y sexualidad en la experiencia estudiantil a partir de relatos y observación realizada en las dos facultades mencionadas. De esta manera, las diferencias entre cada una de las facultades nombran un conjunto de características que adquieren los itinerarios biográficos en tanto formas habituales de atravesar la vida cotidiana en la institución.

Esa vinculación con las formas institucionales propias de cada facultad funcionaron como emergente inicial, pero a medida que el trabajo etnográfico en el terreno fue tejiendo una malla más densa, cobraron fuerza otras dimensiones.

Tal como se esgrime en la introducción del libro, Universidad intima y sexualidades públicas es un juego de palabras que busca potenciar lo que en principio no resulta obvio: que las universidades son un espacio de gestión de la identidad personal, y que una de las gestiones habituales que realizan quienes las habitan refiere a los modos correctos, esperables o legítimos de asumir, encarnar y expresar allí el género y la sexualidad según un conjunto de prescripciones y de orientaciones implícitas (Blanco, 2014, p. 13).

Como se puede advertir, la obra no refiere únicamente 
a las diferencias institucionales, sino que también se permite problematizar los alcances de lo público y lo privado en la universidad, reconstruye las dinámicas socio sexuales que se producen en espacios en donde tienen lugar las experiencias cotidianas y privadas de las y los estudiantes, analiza cómo los modos de sociabilidad entre pares impactan en las gestiones de la identidad y reconstruye las agendas y debates sobre el género y la sexualidad en el movimiento estudiantil.

\section{De lo público y lo privado}

Estamos profundamente acostumbrados a referirnos a las instituciones de educación superior argentinas por su carácter público: hay una tradición orgullosa de dicha usanza, pero también un uso de ese significante que no remite a todo lo que se podría representar como público, sino que hay ciertas formas de "lo público y lo privado" que se habitan a partir de una construcción histórica.

En relación a este punto, una entrevista citada en el libro refiere a las distintas formas de visibilidad del relacionamiento afectivo y sexual que predominan en las facultades. Una estudiante dice: "las parejas se ven por todos lados" y en esa frase se condensa la fuerza de una presencia.

Sin embargo, esa presencia no abarca cualquier tipo de modalidad sino que refiere implícitamente a los vínculos heterosexuales. Paralelamente se observa escrita en el baño de la Facultad de Psicología la frase ya citada al inicio de esta reseña: “¿dónde están las lesbianas en esta facultad?". La pregunta evoca mucho más que un simple interrogante. El anonimato del que 
escribe, el espacio en donde fue inscripto (el baño), son elementos que hablan de ciertas modalidades subterráneas, ciertos códigos restringidos de reconocimiento que emergen no públicamente, como los vínculos heterosexuales, sino en la intimidad.

La circulación de las opiniones traducidas en relación a los saberes disciplinares de cada facultad, la "distribución diferencial de los espacios de deseo", las formas de tipificación entre pares, las clasificaciones que moviliza la vestimenta, son solo algunos de los ejes que se analizan en el libro y que se podrían condensar en lo que el autor denomina los umbrales diferenciales de la intimidad pública.

De esta manera Universidad intima y sexualidades públicas. La gestión de la identidad en la experiencia estudiantil es una propuesta de lectura atractiva para aquellos interesados en las complejas articulaciones entre género y sexualidad en un espacio poco abordado, como es el de la universidad pública argentina.

Santos Sharpe, Andrés Becario doctoral Consejo Nacional de Investigaciones Científicas y Técnicas. Instituto de Investigaciones Gino Germani, Facultad de Ciencias Sociales, Universidad de Buenos Aires

\section{Bibliografía}

Carli, S. (2012). El estudiante universitario: hacia una historia del presente de la educación pública. Buenos Aires: Siglo XXI.

Dubet, F. (2006). El declive de la institución: profesiones, sujetos e individuos en la modernidad. Barcelona: Gedisa.

Krotsch, P. (2014). "Los universitarios como actores de reformas en América Latina: ¿han muerto los movimientos estudiantiles?”. En: S. Carli (dir. y comp.), Universidad pública y experiencia estudiantil. Historia, política y vida cotidiana. Buenos Aires: Miño y Dávila editores. 